\title{
DIMENSION IN THE REALM OF TRANSSERIES
}

\author{
MATTHIAS ASCHENBRENNER, LOU VAN DEN DRIES, AND JORIS VAN DER HOEVEN
}

\begin{abstract}
Let $\mathbb{T}$ be the differential field of transseries. We establish some basic properties of the dimension of a definable subset of $\mathbb{T}^{n}$, also in relation to its codimension in the ambient space $\mathbb{T}^{n}$. The case of dimension 0 is of special interest, and can be characterized both in topological terms (discreteness) and in terms of the Herwig-Hrushovski-Macpherson notion of co-analyzability.
\end{abstract}

\section{INTRODUCTION}

The field of Laurent series with real coefficients comes with a natural derivation but is too small to be closed under integration and exponentiation. These defects are cured by passing to a certain canonical extension, the ordered differential field $\mathbb{T}$ of transseries. Transseries are formal series in an indeterminate $x>\mathbb{R}$, such as

$$
\begin{gathered}
-3 \mathrm{e}^{\mathrm{e}^{x}}+\mathrm{e}^{\frac{\mathrm{e}^{x}}{\log x}+\frac{\mathrm{e}^{x}}{\log ^{2} x}+\frac{\mathrm{e}^{x}}{\log ^{3} x}+\cdots}-x^{11}+7 \\
+\frac{\pi}{x}+\frac{1}{x \log x}+\frac{1}{x \log ^{2} x}+\frac{1}{x \log ^{3} x}+\cdots \\
+\frac{2}{x^{2}}+\frac{6}{x^{3}}+\frac{24}{x^{4}}+\frac{120}{x^{5}}+\frac{720}{x^{6}}+\cdots \\
+\mathrm{e}^{-x}+2 \mathrm{e}^{-x^{2}}+3 \mathrm{e}^{-x^{3}}+4 \mathrm{e}^{-x^{4}}+\cdots,
\end{gathered}
$$

where $\log ^{2} x:=(\log x)^{2}$, etc. Transseries, that is, elements of $\mathbb{T}$, are also the logarithmic-exponential series (LE-series, for short) from [5]; we refer to that paper, or to Appendix A of our book [1], for a detailed construction of $\mathbb{T}$.

What we need for now is that $\mathbb{T}$ is a real closed field extension of the field $\mathbb{R}$ of real numbers and that $\mathbb{T}$ comes equipped with a distinguished element $x>\mathbb{R}$, an exponential operation $\exp : \mathbb{T} \rightarrow \mathbb{T}$ and a distinguished derivation $\partial: \mathbb{T} \rightarrow \mathbb{T}$. The exponentiation here is an isomorphism of the ordered additive group of $\mathbb{T}$ onto the ordered multiplicative group $\mathbb{T}^{>}$of positive elements of $\mathbb{T}$. The derivation $\partial$ comes from differentiating a transseries termwise with respect to $x$, and we set $f^{\prime}:=\partial(f)$, $f^{\prime \prime}:=\partial^{2}(f)$, and so on, for $f \in \mathbb{T}$; in particular, $x^{\prime}=1$, and $\partial$ is compatible with exponentiation: $\exp (f)^{\prime}=f^{\prime} \exp (f)$ for $f \in \mathbb{T}$. Moreover, the constant field of $\mathbb{T}$ is $\mathbb{R}$, that is, $\left\{f \in \mathbb{T}: f^{\prime}=0\right\}=\mathbb{R}$; see again [1] for details.

In Section 1 we define for any differential field $K$ (of characteristic 0 in this paper) and any set $S \subseteq K^{n}$ its (differential-algebraic) dimension

$$
\operatorname{dim} S \in\{-\infty, 0,1, \ldots, n\} \quad(\text { with } \operatorname{dim} S=-\infty \text { iff } S=\emptyset) .
$$

Date: December 2016.

2010 Mathematics Subject Classification. Primary: 12H05, 12J25; secondary: 03C64, 12J15. 
Some dimension properties hold in this generality, but for more substantial results we assume that $K=\mathbb{T}$ and $S$ is definable in $\mathbb{T}$, in which case we have:

$$
\operatorname{dim} S=n \Longleftrightarrow S \text { has nonempty interior in } \mathbb{T}^{n} \text {. }
$$

Here $\mathbb{T}$ is equipped with its order topology, and each $\mathbb{T}^{n}$ with the corresponding product topology. This equivalence is shown in Section 3, where we also prove:

Theorem 0.1. If $S \subseteq \mathbb{T}^{m}$ and $f: S \rightarrow \mathbb{T}^{n}$ are definable, then $\operatorname{dim} S \geqslant \operatorname{dim} f(S)$, for every $i \in\{0, \ldots, m\}$ the set $B(i):=\left\{y \in \mathbb{T}^{n}: \operatorname{dim} f^{-1}(y)=i\right\}$ is definable, and $\operatorname{dim} f^{-1}(B(i))=i+\operatorname{dim} B(i)$.

In Section 4 we show that for definable nonempty $S \subseteq \mathbb{T}^{n}$,

$$
\operatorname{dim} S=0 \Longleftrightarrow S \text { is discrete. }
$$

For $S \subseteq \mathbb{T}^{n}$ to be discrete means as usual that every point of $S$ has a neighborhood in $\mathbb{T}^{n}$ that contains no other point of $S$. For example, $\mathbb{R}^{n}$ as a subset of $\mathbb{T}^{n}$ is discrete! Proving the backwards direction of the equivalence above involves an unusual cardinality argument. Both directions use key results from [1].

The rest of the paper is inspired by [1, Theorem 16.0.3], which suggests that for a definable set $S \subseteq \mathbb{T}^{n}$ to have dimension 0 amounts to $S$ being controlled in some fashion by the constant field $\mathbb{R}$. In what fashion? Our first guess was that perhaps every definable subset of $\mathbb{T}^{n}$ of dimension 0 is the image of some definable map $\mathbb{R}^{m} \rightarrow \mathbb{T}^{n}$. (Every such image has indeed dimension 0.) It turns out, however, that the solution set of the algebraic differential equation $y y^{\prime \prime}=\left(y^{\prime}\right)^{2}$ in $\mathbb{T}$, which has dimension 0, is not such an image: in Section 5 we show how this follows from a fact about automorphisms of $\mathbb{T}$ to be established in [2]. (In that section we call an image as above parametrizable by constants; we have since learned that it already has a name in the literature, namely, internal to the constants, a special case of a general model-theoretic notion; see [14, Section 7.3].)

The correct way to understand the model-theoretic meaning of dimension 0 is the concept of co-analyzability from [8]. This is the topic of Section 6, where we also answer positively a question that partly motivated our paper: given definable $S \subseteq \mathbb{T}^{m}$ and definable $f: S \rightarrow \mathbb{T}^{n}$, does there always exist an $e \in \mathbb{N}$ such that $\left|f^{-1}(y)\right| \leqslant e$ for all $y \in \mathbb{T}^{n}$ for which $f^{-1}(y)$ is finite? In other words, is the quantifier "there exist infinitely many" available for free?

We thank James Freitag for pointing us to the notion of co-analyzability.

\section{Differential-Algebraic Dimension}

We summarize here parts of subsection 2.25 in [4], referring to that paper for proofs. Throughout this section $K$ is a differential field (of characteristic zero with a single distinguished derivation, in this paper), with constant field $C \neq K$. Also, $Y=\left(Y_{1}, \ldots, Y_{n}\right)$ is a tuple of distinct differential indeterminates, and $K\{Y\}$ the ring of differential polynomials in $Y$ over $K$.

Generalities. Let a set $S \subseteq K^{n}$ be given. Then the differential polynomials $P_{1}, \ldots, P_{m} \in K\{Y\}$ are said to be d-algebraically dependent on $S$ if for some nonzero differential polynomial $F \in K\left\{X_{1}, \ldots, X_{m}\right\}$,

$$
F\left(P_{1}(y), \ldots, P_{m}(y)\right)=0 \text { for all } y=\left(y_{1}, \ldots, y_{n}\right) \in S
$$


if no such $F$ exists, we say that $P_{1}, \ldots, P_{m}$ are d-algebraically independent on $S$, and in that case we must have $m \leqslant n$; the prefix d stands for differential. For nonempty $S$ we define the (differential-algebraic) dimension $\operatorname{dim} S$ of $S$ to be the largest $m$ for which there exist $P_{1}, \ldots, P_{m} \in K\{Y\}$ that are d-algebraically independent on $S$, and if $S=\emptyset$, then we set $\operatorname{dim} S:=-\infty$.

In particular, for nonempty $S, \operatorname{dim} S=0$ means that for every $P \in K\{Y\}$ there exists a nonzero $F \in K\{X\}, X=X_{1}$, such that $F(P(y))=0$ for all $y \in S$. As an example, let $a \in K^{n}$ and consider $S=\{a\}$. For $P \in K\{Y\}$ we have $F(P(a))=0$ for $F(X):=X-P(a)$, so $\operatorname{dim}\{a\}=0$. Also, $\operatorname{dim} C^{n}=0$ by Lemma 1.1.

Of course, this notion of dimension is relative to $K$, and if we need to indicate the ambient $K$ we write $\operatorname{dim}_{K} S$ instead of $\operatorname{dim} S$. But this will hardly be necessary, since $\operatorname{dim}_{K} S=\operatorname{dim}_{L} S$ for any differential field extension $L$ of $K$.

Below we also consider the structure $(K, S)$ : the differential field $K$ equipped with the $n$-ary relation $S$. The following is a useful characterization of dimension in terms of differential transcendence degree (for which see [1, Section 4.1]):

Lemma 1.1. Let $\left(K^{*}, S^{*}\right)$ be a $|K|^{+}$-saturated elementary extension of $(K, S)$ and assume $S$ is not empty. Then

$\operatorname{dim}_{K} S=\max \left\{\right.$ differential transcendence degree of $K\langle s\rangle$ over $\left.K: s \in S^{*}\right\}$.

Here are some easy consequences of the definition of dimension and Lemma 1.1:

Lemma 1.2. Let $S, S_{1}, S_{2} \subseteq K^{n}$. Then:

(i) if $S$ is finite and nonempty, then $\operatorname{dim} S=0$; $\operatorname{dim} K^{n}=n$;

(ii) $\operatorname{dim} S<n \Longleftrightarrow S \subseteq\left\{y \in K^{n}: P(y)=0\right\}$ for some nonzero $P \in K\{Y\}$;

(iii) $\operatorname{dim}\left(S_{1} \cup S_{2}\right)=\max \left(\operatorname{dim} S_{1}, \operatorname{dim} S_{2}\right)$;

(iv) $\operatorname{dim} S^{\sigma}=\operatorname{dim} S$ for each permutation $\sigma$ of $\{1, \ldots, n\}$, where

$$
S^{\sigma}:=\left\{\left(y_{\sigma(1)}, \ldots, y_{\sigma(n)}\right):\left(y_{1}, \ldots, y_{n}\right) \in S\right\} ;
$$

(v) if $m \leqslant n$ and $\pi: K^{n} \rightarrow K^{m}$ is given by $\pi\left(y_{1}, \ldots, y_{n}\right)=\left(y_{1}, \ldots, y_{m}\right)$, then $\operatorname{dim} \pi(S) \leqslant \operatorname{dim} S$

(vi) if $\operatorname{dim} S=m$, then $\operatorname{dim} \pi\left(S^{\sigma}\right)=m$ for some $\sigma$ as in (iv) and $\pi$ as in (v).

The next two lemmas are not in [4], and are left as easy exercises:

Lemma 1.3. $\operatorname{dim}\left(S_{1} \times S_{2}\right)=\operatorname{dim} S_{1}+\operatorname{dim} S_{2}$ for $S_{1} \subseteq K^{m}$ and $S_{2} \subseteq K^{n}$.

Lemma 1.4. $\operatorname{dim}_{K} S=\operatorname{dim}_{K^{*}} S^{*}$ in the situation of Lemma 1.1.

Let now $K^{*}$ be any elementary extension of $K$ and suppose $S$ is definable in $K$, say by the formula $\phi\left(y_{1}, \ldots, y_{n}\right)$ in the language of differential fields with names for the elements of $K$. Let $S^{*} \subseteq\left(K^{*}\right)^{n}$ be defined in $K^{*}$ by the same formula $\phi\left(y_{1}, \ldots, y_{n}\right)$. Note that $S^{*}$ does not depend on the choice of $\phi$. We have the following easy consequence of Lemma 1.4:

Corollary 1.5. $\operatorname{dim}_{K} S=\operatorname{dim}_{K^{*}} S^{*}$.

Differential boundedness. For a set $S \subseteq K^{n+1}$ and $y \in K^{n}$ we define

$$
S(y):=\{z \in K:(y, z) \in S\} \quad \text { (the section of } S \text { above } y \text { ). }
$$

We say that $K$ is d-bounded if for every definable set $S \subseteq K^{n+1}$ there exist $P_{1}, \ldots, P_{m} \in K\{Y, Z\}$ (with $Z$ an extra indeterminate) such that if $y \in K^{n}$ and $\operatorname{dim} S(y)=0$, then $S(y) \subseteq\left\{z \in K: P_{i}(y, z)=0\right\}$ for some $i \in\{1, \ldots, m\}$ 
with $P_{i}(y, Z) \neq 0$. (In view of Lemma 1.2(ii), this is equivalent to the differential field $K$ being differentially bounded as defined on p. 203 of [4].) Here is the main consequence of d-boundedness, taken from [4]:

Proposition 1.6. Assume $K$ is d-bounded. Let $S \subseteq K^{m}$ and $f: S \rightarrow K^{n}$ be definable. Then $\operatorname{dim} S \geqslant \operatorname{dim} f(S)$. Moreover, for every $i \in\{0, \ldots, m\}$ the set $B(i):=\left\{y \in K^{n}: \operatorname{dim} f^{-1}(y)=i\right\}$ is definable, and $\operatorname{dim} f^{-1}(B(i))=i+\operatorname{dim} B(i)$.

As $\mathbb{T}$ is d-bounded (see Section 3), this gives Theorem 0.1. Differentially closed fields are d-bounded, as pointed out in [4]. Guzy and Point [7] (see also [3]) show that existentially closed ordered differential fields, and Scanlon's d-henselian valued differential fields with many constants (see [1, Chapter 8]) are d-bounded.

\section{Dimension And Codimension}

This section will not be used in the rest of this paper, but is included for its own sake. The main result is Corollary 2.3. A byproduct of the treatment here is a simpler proof of [1, Theorem 5.9.1] that avoids the nontrivial facts about regular local rings used in [1], where we followed closely Johnson's proof in [10] of a more general result.

Let $y=\left(y_{1}, \ldots, y_{n}\right)$ be a tuple of elements of a differential field extension of $K$, and let $d$ be the differential transcendence degree of $F:=K\langle y\rangle$ over $K$ : there are $i_{1}<\cdots<i_{d}$ in $\{1, \ldots, n\}$ such that $y_{i_{1}}, \ldots, y_{i_{d}}$ are d-algebraically independent over $K$, but there are no $i_{1}<\cdots<i_{d}<i_{d+1}$ in $\{1, \ldots, n\}$ such that $y_{i_{1}}, \ldots, y_{i_{d}}, y_{i_{d+1}}$ are d-algebraically independent over $K$. We wish to characterize $d$ alternatively as follows: there should exist $n-d$ "independent" relations $P_{1}(y)=\cdots=P_{n-d}(y)=0$, with all $P_{i} \in K\{Y\}$, but not more than $n-d$ such relations. The issue here is what "independent" should mean.

We say that a d-polynomial $P \in K\{Y\}$ has order at most $\vec{r}=\left(r_{1}, \ldots, r_{n}\right) \in \mathbb{N}^{n}$ if $P \in K\left[Y_{j}^{(r)}: 1 \leqslant j \leqslant n, 0 \leqslant r \leqslant r_{j}\right]$. Given $P_{1}, \ldots, P_{m} \in K\{Y\}$ of order at most $\vec{r} \in \mathbb{N}^{n}$, consider the $m \times n$-matrix over $F$ with $i, j$-entry

$$
\frac{\partial P_{i}}{\partial Y_{j}^{\left(r_{j}\right)}}(y) \quad(i=1, \ldots, m, j=1, \ldots, n) .
$$

This matrix has rank $\leqslant \min (m, n)$. We say that $P_{1}, \ldots, P_{m}$ are strongly d-independent at $y$ if for some $\vec{r} \in \mathbb{N}^{n}$ with $P_{1}, \ldots, P_{m}$ of order at most $\vec{r}$, this matrix has rank $m$; thus $m \leqslant n$ in that case.

Set $R:=K\{Y\}$ and $\mathfrak{p}:=\{P \in R: P(y)=0\}$, a differential prime ideal of $R$. With these notations we have:

Lemma 2.1. There are $P_{1}, \ldots, P_{n-d} \in \mathfrak{p}$ that are strongly $\mathrm{d}$-independent at $y$.

Proof. Set $m:=n-d$ and permute indices such that $y_{m+1}, \ldots, y_{n}$ is a differential transcendence base of $F=K\langle y\rangle$ over $K$. For $i=1, \ldots, m$, pick

$$
P_{i}\left(Y_{i}, Y_{m+1}, \ldots, Y_{n}\right) \in K\left\{Y_{i}, Y_{m+1}, \ldots, Y_{n}\right\} \subseteq K\{Y\}
$$

such that $P_{i}\left(Y_{i}, y_{m+1}, \ldots, y_{n}\right)$ is a minimal annihilator of $y_{i}$ over $K\left\langle y_{m+1}, \ldots, y_{n}\right\rangle$. Let $P_{i}$ have order $r_{i}$ in $Y_{i}$. Then the minimality of $P_{i}$ gives

$$
\frac{\partial P_{i}}{\partial Y_{i}^{\left(r_{i}\right)}}\left(y_{i}, y_{m+1}, \ldots, y_{n}\right) \neq 0, \quad(i=1, \ldots, m) .
$$


Next we take $r_{m+1}, \ldots, r_{n} \in \mathbb{N}$ such that all $P_{i}$ have order $\leqslant r_{j}$ in $Y_{j}$ for $j=$ $m+1, \ldots, n$. Considering all $P_{i}$ as elements of $K\{Y\}$ we see that $P_{1}, \ldots, P_{m}$ have order $\leqslant\left(r_{1}, \ldots, r_{n}\right)$, and that the $m \times m$ matrix

$$
\left(\frac{\partial P_{i}}{\partial Y_{j}^{\left(r_{j}\right)}}(y)\right) \quad(1 \leqslant i, j \leqslant m)
$$

is diagonal, with nonzero determinant.

We refer to $\left[1\right.$, Section 5.4] for what it means for $P_{1}, \ldots, P_{m} \in R$ to be d-independent at $y$. By [1, Lemma 5.4.7], if $P_{1}, \ldots, P_{m} \in R$ are strongly d-independent at $y$, then they are d-independent at $y$ (but the converse may fail). Below we show that if $P_{1}, \ldots, P_{m} \in \mathfrak{p}$ are d-independent at $y$, then $m \leqslant n-d$.

The notion of d-independence at $y$ is more intrinsic and more flexible than that of strong d-independence at $y$. To discuss the former in more detail, we need some terminology from [1]. Let $A$ be a commutative ring, $\mathfrak{p}$ a prime ideal of $A$, and $M$ an $A$-module; then a family $\left(f_{i}\right)$ of elements of $M$ is said to be independent at $\mathfrak{p}$ if the family $\left(f_{i}+\mathfrak{p} M\right)$ of elements of the $A / \mathfrak{p}$-module $M / \mathfrak{p} M$ is linearly independent. Next, let $A$ also be a differential ring extension of $K$. Then the $K$-algebra $A$ yields the $A$-module $\Omega_{A \mid K}$ of Kähler differentials with the (universal) $K$-derivation

$$
a \mapsto \mathrm{d} a=\mathrm{d}_{A \mid K} a: A \rightarrow \Omega_{A \mid K} .
$$

Following Johnson [10] we make this $A$-module compatibly into an $A[\partial]$-module by $\partial(\mathrm{d} a):=\mathrm{d} \partial a$ for $a \in A$; a family of elements of $\Omega_{A \mid K}$ is said to be d-independent if this family is linearly independent in $\Omega_{A \mid K}$ viewed as an $A[\partial]$-module. This means for $a_{1}, \ldots, a_{m} \in A$ : the differentials $\mathrm{d} a_{1}, \ldots, \mathrm{d} a_{m} \in \Omega_{A \mid K}$ are d-independent iff the family $\left(\mathrm{d} a_{i}^{(r)}\right)(i=1, \ldots, m, r=0,1,2, \ldots)$ is linearly independent in the $A$-module $\Omega_{A \mid K}$; given also a prime ideal $\mathfrak{p}$ of $A$ we say that $\mathrm{d} a_{1}, \ldots, \mathrm{d} a_{m}$ are $\mathrm{d}$-independent at $\mathfrak{p}$ if the family $\left(\mathrm{d} a_{i}^{(r)}\right)$ is independent at $\mathfrak{p}$ in the $A$-module $\Omega_{A \mid K}$.

Returning to the differential ring extensions $R$ and $F=K\langle y\rangle$ of $K$, the $R[\partial]$ module $\Omega_{R \mid K}$ is free on $\mathrm{d} Y_{1}, \ldots, \mathrm{d} Y_{n}$, by [1, Lemma 1.8.11]. The $F[\partial]$-module $\Omega_{F \mid K}$ is generated by $\mathrm{d} y_{1}, \ldots, \mathrm{d} y_{n}$, as shown in [1, Section 5.9]. In [1, Section 5.3] we assign to every finitely generated $F[\partial]$-module $M$ a number $\operatorname{rank}(M) \in \mathbb{N}$, and we have $\operatorname{rank}\left(\Omega_{F \mid K}\right)=d$ by [1, Corollary 5.9.3].

The differential ring morphism $P \mapsto P(y): R \rightarrow F$ is the identity on $K$, and makes $F \otimes_{R} \Omega_{R \mid K}$ into an $F[\partial]$-module as explained in [1, Section 5.9]. Note that the kernel of the above differential ring morphism $R \rightarrow F$ is the differential prime ideal $\mathfrak{p}=\{P \in R: P(y)=0\}$ of $R$.

Lemma 2.2. Suppose $P_{1}, \ldots, P_{m} \in \mathfrak{p}$ are $\mathrm{d}$-independent at $y$. Then $m \leqslant n-d$.

Proof. We have a surjective $F[\partial]$-linear map $F \otimes_{R} \Omega_{R \mid K} \rightarrow \Omega_{F \mid K}$ sending $1 \otimes \mathrm{d} P$ to $\mathrm{d} P(y)$ for $P \in R$. Note that $1 \otimes \mathrm{d} P_{1}, \ldots, 1 \otimes \mathrm{d} P_{m}$ are in the kernel of this map. By the equivalence $(1) \Leftrightarrow(5)$ and Lemma 5.9 .4 in [1], the d-independence of $P_{1}, \ldots, P_{m}$ at $y$ gives that $1 \otimes \mathrm{d} P_{1}, \ldots, 1 \otimes \mathrm{d} P_{m} \in F \otimes_{R} \Omega_{R \mid K}$ are $F[\partial]$-independent (meaning: linearly independent in this $F[\partial]$-module). Since the $R[\partial]$-module $\Omega_{R \mid K}$ is free on $d Y_{1}, \ldots, d Y_{n}$, the $F[\partial]$-module $F \otimes_{R} \Omega_{R \mid K}$ is free on $1 \otimes \mathrm{d} Y_{1}, \ldots, 1 \otimes \mathrm{d} Y_{n}$, and so has rank $n$. To get $m+d \leqslant n$ it remains to use [1, Corollary 5.9.3] and the fact that $\operatorname{rank}\left(\Omega_{F \mid K}\right)=d$.

Combining the previous two lemmas we conclude: 
Corollary 2.3. The codimension $n-d$ can be characterized as follows:

$$
\begin{aligned}
n-d & =\max \left\{m: \text { some } P_{1}, \ldots, P_{m} \in \mathfrak{p} \text { are } \mathrm{d} \text {-independent at } y\right\} \\
& =\max \left\{m: \text { some } P_{1}, \ldots, P_{m} \in \mathfrak{p} \text { are strongly } \mathrm{d} \text {-independent at } y\right\} .
\end{aligned}
$$

This yields a strengthening of Theorem 5.9.1 and its Corollary 5.9.6 in [1]:

Corollary 2.4. The following are equivalent:

(i) $y_{1}, \ldots, y_{n}$ are d-algebraic over $K$;

(ii) there exist $P_{1}, \ldots, P_{n} \in \mathfrak{p}$ that are $\mathrm{d}-$ independent at $y$;

(iii) there exist $P_{1}, \ldots, P_{n} \in \mathfrak{p}$ that are are strongly d-independent at $y$.

To formulate the above in terms of sets $S \subseteq K^{n}$ we recall that the Kolchin topology on $K^{n}$ (called the differential-Zariski topology on $K^{n}$ in [4]) is the topology on $K^{n}$ whose closed sets are the sets

$$
\left\{y \in K^{n}: P_{1}(y)=\cdots=P_{m}(y)=0\right\} \quad\left(P_{1}, \ldots, P_{m} \in K\{Y\}\right) .
$$

This is a noetherian topology, and so a Kolchin closed subset of $K^{n}$ is the union of its finitely many irreducible components. For $S \subseteq K^{n}$ we let $S^{\text {Ko }}$ be its Kolchin closure in $K^{n}$ with respect to the Kolchin topology. Note that $\operatorname{dim} S=\operatorname{dim} S^{\mathrm{Ko}}$, since for all $P \in K\{Y\}$ we have: if $P=0$ on $S$ (that is, $P(y)=0$ for all $y \in S$ ), then $P=0$ on $S^{\mathrm{Ko}}$.

Suppose $S^{\mathrm{Ko}}$ is irreducible. A tuple of $m$ independent relations on $S$ is defined to be a tuple $\left(P_{1}, \ldots, P_{m}\right) \in K\{Y\}^{m}$ such that

(1) $P_{1}(y)=\cdots=P_{m}(y)=0$ for all $y \in S$;

(2) $P_{1}, \ldots, P_{m}$ are d-independent at some $y \in S$.

Similarly we define a tuple of $m$ strongly independent relations on $S$, by replacing "d-independent" in (2) by "strongly d-independent". Every tuple of strongly independent relations on $S$ is a tuple of independent relations on $S$. Since $S^{\mathrm{Ko}}$ is irreducible,

$$
\mathfrak{p}:=\{P \in K\{Y\}: P=0 \text { on } S\}
$$

is a differential prime ideal of $K\{Y\}$. Letting $K\{y\}=K\{Y\} / \mathfrak{p}$ be the corresponding differential $K$-algebra (an integral domain) with $y=\left(y_{1}, \ldots, y_{n}\right), y_{i}=Y_{i}+\mathfrak{p}$, for $P \in K\{Y\}$ we have $P(y)=0$ iff $P=0$ on $S$. So the considerations above applied to $y$ yield for $d:=\operatorname{dim} S$ and irreducible $S^{\mathrm{Ko}}$ :

Corollary 2.5. There is a tuple of $m$ strongly independent relations on $S$ for $m=n-d$, but there is no tuple of $m$ independent relations on $S$ for $m>n-d$.

\section{The CASe of $\mathbb{T}$}

The paper [4] contains an axiomatic framework for a reasonable notion of dimension for the definable sets in suitable model-theoretic structures with a topology. In this section we show that as a consequence of [1, Chapter 16] the relevant axioms are satisfied for $\mathbb{T}$ with its order topology.

To state the necessary facts about $\mathbb{T}$ from [1] we recall from that book that an $H$-field is an ordered differential field $K$ with constant field $C$ such that:

(H1) $\partial(a)>0$ for all $a \in K$ with $a>C$;

(H2) $\mathcal{O}=C+\mathcal{O}$, where $\mathcal{O}$ is the convex hull of $C$ in the ordered field $K$, and $\mathcal{O}$ is the maximal ideal of the valuation ring $\mathcal{O}$. 
Let $K$ be an $H$-field, and let $\mathcal{O}$ and $\mathcal{O}$ be as in (H2). Thus $K$ is a valued field with valuation $\operatorname{ring} \mathcal{O}$. The valuation topology on $K$ equals its order topology if $C \neq K$. We consider $K$ as an $\mathcal{L}$-structure, where

$$
\mathcal{L}:=\{0,1,+,-, \times, \partial, P, \preccurlyeq\}
$$

is the language of ordered valued differential fields. The symbols $0,1,+,-, \times, \partial$ are interpreted as usual in $K$, and $P$ and $\preccurlyeq$ encode the ordering and the valuation:

$$
P(a) \Longleftrightarrow a>0, \quad a \preccurlyeq b \Longleftrightarrow a \in \mathcal{O} b \quad(a, b \in K) .
$$

Given $a \in K$ we also write $a^{\prime}$ instead of $\partial(a)$, and we set $a^{\dagger}:=a^{\prime} / a$ for $a \neq 0$.

The real closed (and thus ordered) differential field $\mathbb{T}$ is an $H$-field, and in [1] we showed that it is a model of a model-complete $\mathcal{L}$-theory $T^{\mathrm{nl}}$. The models of the latter are exactly the $H$-fields $K$ satisfying the following (first-order) conditions:

(1) $K$ is Liouville closed;

(2) $K$ is $\omega$-free;

(3) $K$ is newtonian.

(An $H$-field $K$ is said to be Liouville closed if it is real closed and for all $a \in K$ there exists $b \in K$ with $a=b^{\prime}$ and also a $b \in K^{\times}$such that $a=b^{\dagger}$; for the definition of " $\omega$-free" and "newtonian" we refer to the Introduction of [1].) Since "Liouville closed" includes "real closed", the ordering (and thus the valuation ring) of any model of $T^{\mathrm{nl}}$ is definable in the underlying differential field of the model. We shall prove the dimension results in this paper for all models of $T^{\mathrm{nl}}$ : working in this generality plays a role even when our main interest is in $\mathbb{T}$. So in the rest of this section we fix an arbitrary model $K$ of $T^{\mathrm{nl}}$, that is, $K$ is a Liouville closed $\omega$-free newtonian $H$-field. Lemma 1.2(ii) and [1, Corollary 16.6.4] yield:

Corollary 3.1. For definable $S \subseteq K^{n}$,

$$
\operatorname{dim} S=n \Longleftrightarrow S \text { has nonempty interior in } K^{n} .
$$

To avoid confusion with the Kolchin topology, we consider $K$ here and below as equipped with its order topology, and $K^{n}$ with the corresponding product topology. Combining the previous corollary with (iv)-(vi) in Lemma 1.2 yields a topological characterization of dimension:

Corollary 3.2. For nonempty definable $S \subseteq K^{n}$, $\operatorname{dim} S$ is the largest $m \leqslant n$ such that for some permutation $\sigma$ of $\{1, \ldots, n\}$, the subset $\pi_{m}\left(S^{\sigma}\right)$ of $K^{m}$ has nonempty interior; here $\pi_{m}\left(x_{1}, \ldots, x_{n}\right):=\left(x_{1}, \ldots, x_{m}\right)$ for $\left(x_{1}, \ldots, x_{n}\right) \in K^{n}$.

In particular, if $S \subseteq K^{n}$ is semialgebraic in the sense of the real closed field $K$, then $\operatorname{dim} S$ agrees with the usual semialgebraic dimension of $S$ over $K$.

To get that $K$ is d-bounded, we introduce two key subsets of $K$, namely $\Lambda(K)$ and $\Omega(K)$. They are defined by the following equivalences, for $a \in K$ :

$$
\begin{aligned}
& a \in \Lambda(K) \Longleftrightarrow a=-y^{\dagger \dagger} \text { for some } y \succ 1 \text { in } K, \\
& a \in \Omega(K) \Longleftrightarrow 4 y^{\prime \prime}+a y=0 \text { for some } y \in K^{\times} .
\end{aligned}
$$


To describe these sets more concretely for $K=\mathbb{T}$, set $\ell_{0}:=x$ and $\ell_{n+1}:=\log \ell_{n}$, so $\ell_{n}$ is the $n$th iterated logarithm of $x$ in $\mathbb{T}$. Then for $f \in \mathbb{T}$,

$$
\begin{aligned}
& f \in \Lambda(\mathbb{T}) \Longleftrightarrow f<\frac{1}{\ell_{0}}+\frac{1}{\ell_{0} \ell_{1}}+\cdots+\frac{1}{\ell_{0} \ell_{1} \cdots \ell_{n}} \quad \text { for some } n, \\
& f \in \Omega(\mathbb{T}) \Longleftrightarrow f<\frac{1}{\ell_{0}^{2}}+\frac{1}{\ell_{0}^{2} \ell_{1}^{2}}+\cdots+\frac{1}{\ell_{0}^{2} \ell_{1}^{2} \cdots \ell_{n}^{2}} \quad \text { for some } n,
\end{aligned}
$$

by [1, Example after 11.8.19; Proposition 11.8.20 and Corollary 11.8.21]. The set $\Lambda(K)$ is closed downward in $K$ : if $a \in K$ and $a<b \in \Lambda(K)$, then $a \in \Lambda(K)$; and $\Lambda(K)$ has an upper bound in $K$ but no least upper bound; these properties also hold for $\Omega(K)$ instead of $\Lambda(K)$. From Chapter 16 of [1] we need that $T^{\mathrm{nl}}$ has a certain extension by definitions $T_{\Lambda \Omega}^{\text {nl }}$ that has QE: the language of $T_{\Lambda \Omega}^{\text {nl }}$ is $\mathcal{L}$ augmented by two extra binary relation symbols $R_{\Lambda}$ and $R_{\Omega}$, to be interpreted in $K$ according to

$$
a R_{\Lambda} b \Longleftrightarrow a \in \Lambda(K) b, \quad a R_{\Omega} b \Longleftrightarrow a \in \Omega(K) b .
$$

(The language of $T_{\Lambda \Omega}^{\mathrm{nl}}$ in [1, Chapter 16] is slightly different, but yields the same notion of what is quantifier-free definable. The version here is more convenient for our purpose.) Using that $\Lambda(K)$ and $\Omega(K)$ are open-and-closed in $K$, it is routine (but tedious) to check that $K$ satisfies the differential analogue of $[4,2.15]$ that is discussed on p. 203 of that paper in a general setting. Thus:

Corollary 3.3. $K$ is d-bounded; in particular, $\mathbb{T}$ is d-bounded.

Moreover, [4, p. 203] points out the following consequence (extending Corollary 3.1):

Corollary 3.4. Every nonempty definable set $S \subseteq K^{n}$ has nonempty interior in the Kolchin closure $S^{\mathrm{Ko}}$ of $S$ in $K^{n}$.

(By our earlier convention, the interior here refers to the topology on $S^{\mathrm{Ko}}$ induced by the product topology on $K^{n}$ that comes from the order topology on $K$.) For nonempty definable $S \subseteq K^{n}$ with closure $\operatorname{cl}(S)$ in $K^{n}$ we have

$$
\operatorname{dim}(\operatorname{cl}(S) \backslash S)<\operatorname{dim} S .
$$

This is analogous to $[4,2.23]$, but the proof there doesn't go through. We intend to show this dimension decrease in a follow-up paper.

\section{Dimension $0=$ Discrete}

Let $K$ be a Liouville closed $\omega$-free newtonian $H$-field, with the order topology on $K$ and the corresponding product topology on each $K^{n}$. Corollary 16.6.11 in [1] and its proof yields the following equivalences for definable $S \subseteq K$ :

$$
\operatorname{dim} S=0 \Longleftrightarrow S \text { has empty interior } \Longleftrightarrow S \text { is discrete. }
$$

We now extend part of this to definable subsets of $K^{n}$. The proof of one of the directions is rather curious and makes full use of the resources of [1].

Proposition 4.1. For definable nonempty $S \subseteq K^{n}$ :

$$
\operatorname{dim} S=0 \Longleftrightarrow S \text { is discrete. }
$$


Proof. For $i=1, \ldots, n$ we let $\pi_{i}: K^{n} \rightarrow K$ be given by $\pi_{i}\left(a_{1}, \ldots, a_{n}\right)=a_{i}$. If $\operatorname{dim} S=0$, then $\operatorname{dim} \pi_{i}(S)=0$ for all $i$, so $\pi_{i}(S)$ is discrete for all $i$, hence the cartesian product $\pi_{1}(S) \times \cdots \times \pi_{n}(S) \subseteq K^{n}$ is discrete, and so is its subset $S$.

Now for the converse. Assume $S \subseteq K^{n}$ is discrete. We first replace $K$ by a suitable countable elementary substructure over which $S$ is defined and $S$ by its corresponding trace. Now that $K$ is countable we next pass to its completion $K^{\mathrm{c}}$ as defined in $[1$, Section 4.4], which by $[1,14.1 .6]$ is an elementary extension of $K$. Replacing $K$ by $K^{\mathrm{c}}$ and $S$ by the corresponding extension, the overall effect is that we have arranged $K$ to be uncountable, but with a countable base for its topology. Then the discrete set $S$ is countable, so $\pi_{i}(S) \subseteq K$ is countable for each $i$, hence with empty interior, so $\operatorname{dim} \pi_{i}(S)=0$ for all $i$, and thus $\operatorname{dim} S=0$.

Corollary 4.2. If $S \subseteq K^{n}$ is definable and discrete, then there is a neighborhood $U$ of $0 \in K^{n}$ such that $\left(s_{1}+U\right) \cap\left(s_{2}+U\right)=\emptyset$ for all distinct $s_{1}, s_{2} \in S$.

Proof. Let $S \subseteq K^{n}(n \geqslant 1)$ be nonempty, definable, and discrete. For $y \in K^{n}$ we set $|y|:=\max _{i}\left|y_{i}\right|$. The set $D:=\{|a-b|: a, b \in S\}$ is the image of a definable map $S^{2} \rightarrow K$, so $D$ is definable with $\operatorname{dim} D=0$ and $0 \in D$. Thus $D$ is discrete, so $(-\varepsilon, \varepsilon) \cap D=\{0\}$ for some $\varepsilon \in K^{>}$, which gives the desired conclusion.

In particular, any definable discrete subset of $K^{n}$ is closed in $K^{n}$.

\section{Parametrizability by Constants}

Let $K$ be a Liouville closed $\omega$-free newtonian $H$-field. Then $K$ induces on its constant field $C$ just $C$ 's structure as a real closed field, by [1, 16.0.2(ii)], that is, a set $X \subseteq C^{m}$ is definable in $K$ iff $X$ is semialgebraic in the sense of $C$.

Let $S \subseteq K^{n}$ be definable. We say that $S$ is parametrizable by constants if $S \subseteq f\left(\bar{C}^{m}\right)$ for some $m$ and some definable map $f: C^{m} \rightarrow K^{n}$; equivalently, $S=f(X)$ for some injective definable map $f: X \rightarrow K^{n}$ with semialgebraic $X \subseteq C^{m}$ for some $m$. (The reduction to injective $f$ uses the fact mentioned above about the induced structure on $C$.) For example, if $P \in K\{Y\}$ is a differential polynomial of degree 1 in a single indeterminate $Y$, then the set $\{y \in K: P(y)=0\}$ is either empty or a translate of a finite-dimensional $C$-linear subspace of $K$, and so this set is parametrizable by constants. The definable sets in $K^{n}$ for $n=0,1,2, \ldots$ that are parametrizable by constants make up a very robust class: it is closed under taking definable subsets, and under some basic logical operations: taking finite unions (in the same $K^{n}$ ), cartesian products, and images under definable maps. Moreover:

Lemma 5.1. Let $S \subseteq K^{n}$ and $f: S \rightarrow C^{m}$ be definable, and let $e \in \mathbb{N}$ be such that $\left|f^{-1}(c)\right| \leqslant e$ for all $c \in C^{m}$. Then $S$ is parametrizable by constants.

Proof. By partitioning $S$ appropriately we reduce to the case that for all $c \in f(S)$ we have $\left|f^{-1}(c)\right|=e$. Using the lexicographic ordering on $K^{n}$ this yields definable injective $g_{1}, \ldots, g_{e}: f(S) \rightarrow K^{n}$ such that $f^{-1}(c)=\left\{g_{1}(c), \ldots, g_{e}(c)\right\}$ for all $c \in$ $f(S)$. Thus $S=g_{1}(f(S)) \cup \cdots \cup g_{e}(f(S))$ is parametrizable by constants.

Suppose $S \subseteq K^{n}$ be definable. Note that if $S$ is parametrizable by constants, then $\operatorname{dim} S \leqslant 0$. The question arises if the converse holds: does it follow from $\operatorname{dim} S=0$

that $S$ is parametrizable by constants? We show that the answer is negative for $K=\mathbb{T}$ and the set

$$
\left\{y \in \mathbb{T}: y y^{\prime \prime}=\left(y^{\prime}\right)^{2}\right\}=\left\{a \mathrm{e}^{b x}: a, b \in \mathbb{R}\right\} .
$$


This set has dimension 0 and we claim that it is not parametrizable by constants. (The map $(a, b) \mapsto a \mathrm{e}^{b x}: \mathbb{R}^{2} \rightarrow \mathbb{T}$ would be a parametrization of this set by constants if exp were definable in $\mathbb{T}$; we return to this issue at the end of this section.) To justify this claim we appeal to a special case of results from [2]:

For any finite set $A \subseteq \mathbb{T}$ there exists an automorphism of the differential field $\mathbb{T}$ over $A$ that is not the identity on $\left\{\mathrm{e}^{b x}: b \in \mathbb{R}\right\}$.

The claimed nonparametrizability by constants follows when we combine this fact with the observation that if $f: \mathbb{R}^{m} \rightarrow \mathbb{T}$ is definable in $\mathbb{T}$, say over the finite set $A \subseteq \mathbb{T}$, then any automorphism of the differential field $\mathbb{T}$ over $A$ fixes each real number, and so it fixes each value of the function $f$.

Below $Y$ is a single indeterminate, and for $P \in K\{Y\}$ we let

$$
\mathrm{Z}(P):=\{y \in K: P(y)=0\} .
$$

Thus $\mathrm{Z}\left(Y Y^{\prime \prime}-\left(Y^{\prime}\right)^{2}\right)=\left\{a \mathrm{e}^{b x}: a, b \in \mathbb{R}\right\}$ for $K=\mathbb{T}$ and $Y Y^{\prime \prime}-\left(Y^{\prime}\right)^{2}$ has order 2 . What about the parametrizability of $\mathrm{Z}(P)$ for $P$ of order 1 ? In the next two lemmas we consider the special case $P(Y)=F(Y) Y^{\prime}-G(Y)$ where $F, G \in C[Y]^{\neq}$have no common factor of positive degree.

Lemma 5.2. If $\frac{F}{G}=c \frac{\partial R}{\partial Y} / R$ for some $c \in C^{\times}, R \in C(Y)^{\times}$, or $\frac{F}{G}=\frac{\partial R}{\partial Y}$ for some $R \in C(Y)^{\times}$, then $\mathrm{Z}(P)$ is parametrizable by constants.

Proof. Suppose $\frac{F}{G}=c \frac{\partial R}{\partial Y} / R$ where $c \in C^{\times}, R \in C(Y)^{\times}$. Since $K$ is Liouville closed we can take $b \in K^{\times}$with $b^{\dagger}=1 / c$. Set $S:=\{y \in \mathrm{Z}(P): G(y) \neq 0, R(y) \neq 0, \infty\}$. Then for $y \in S$ we have

$$
0=G(y)\left(\frac{F(y)}{G(y)} y^{\prime}-1\right)=G(y)\left(c\left(\frac{\partial R}{\partial Y} / R\right)(y) y^{\prime}-1\right)=G(y)\left(c R(y)^{\dagger}-1\right)
$$

and so $R(y) \in C^{\times} b$. It is clear that we can take $e \in \mathbb{N}$ such that the definable map $f: S \rightarrow C$ given by $f(y):=R(y) / b$ for $y \in S$ satisfies $\left|f^{-1}(c)\right| \leqslant e$ for all $c \in C$. Hence $S$, and thus $\mathrm{Z}(P)$, is parametrizable by constants by Lemma 5.1. Next, suppose that $\frac{F}{G}=\frac{\partial R}{\partial Y}$ where $R \in C(Y)$. Take $x \in K$ with $x^{\prime}=1$ and set $S:=\{y \in \mathrm{Z}(P): G(y) \neq 0, R(y) \neq \infty\}$. As before we obtain for $y \in S$ that $R(y) \in x+C$, and so $\mathrm{Z}(P)$ is parametrizable by constants.

Let $Q \in K\{Y\}$ be irreducible and let $a$ be an element of a differential field extension of $K$ with minimal annihilator $Q$ over $K$. We say that $Q$ creates a constant if $C_{K\langle a\rangle} \neq C$. (This is related to the concept of "nonorthogonality to the constants" in the model theory of differential fields; see [12, Proposition 2.6].) Note that our $P=F(Y) Y^{\prime}-G(Y)$ is irreducible in $K\{Y\}$.

Lemma 5.3. $P$ creates a constant iff $\frac{F}{G}=c \frac{\partial R}{\partial Y} / R$ for some $c \in C^{\times}, R \in C(Y)^{\times}$, or $\frac{F}{G}=\frac{\partial R}{\partial Y}$ for some $R \in C(Y)^{\times}$.

Proof. The forward direction holds by Rosenlicht [15, Proposition 2]. For the backward direction, take an element $a$ of a differential field extension of $K$ with minimal annihilator $P$ over $K$. Consider first the case $\frac{F}{G}=c \frac{\partial R}{\partial Y} / R$ where $c \in C^{\times}$ and $R \in C(Y)^{\times}$. Take $b \in K^{\times}$with $b^{\dagger}=1 / c$. As in the proof of Lemma 5.2 we obtain $0=P(a)=G(a)\left(c R(a)^{\dagger}-1\right)$ with $G(a) \neq 0$, and thus $R(a) / b \in C_{K\langle a\rangle}$ and $R(a) / b \notin K$. The case $\frac{F}{G}=\frac{\partial R}{\partial Y}$ with $R \in C(Y)^{\times}$is handled likewise.

The following proposition therefore generalizes Lemma 5.2: 
Proposition 5.4. If $P \in K\{Y\}$ is irreducible of order 1 and creates a constant, then $\mathrm{Z}(P)$ is parametrizable by constants.

Before we give the proof of this proposition, we prove two lemmas, in both of which we let $P \in K\{Y\}$ be irreducible of order 1 such that $\mathrm{Z}(P)$ is infinite.

Lemma 5.5. Let $Q \in K\left[Y, Y^{\prime}\right] \subseteq K\{Y\}$. Then $\mathrm{Z}(P) \subseteq \mathrm{Z}(Q)$ iff $Q \in P K\left[Y, Y^{\prime}\right]$.

Proof. Suppose $\mathrm{Z}(P) \subseteq \mathrm{Z}(Q)$ but $Q \notin P K\left[Y, Y^{\prime}\right]$. Put $F:=K(Y)$. By Gauss' Lemma, $P$ viewed as element of $F\left[Y^{\prime}\right]$ is irreducible and $Q \notin P F\left[Y^{\prime}\right]$. Thus there are $A, B \in K\left[Y, Y^{\prime}\right], D \in K[Y]^{\neq}$with $D=A P+B Q$. Then $\mathrm{Z}(P) \subseteq \mathrm{Z}(D)$ is finite, a contradiction.

Lemma 5.6. There is an element $a$ in an elementary extension of $K$ with minimal annihilator $P$ over $K$.

Proof. Given $Q_{1}, \ldots, Q_{n} \in K\left[Y, Y^{\prime}\right]^{\neq}$with $\operatorname{deg}_{Y^{\prime}} Q_{i}<\operatorname{deg}_{Y^{\prime}} P$ for $i=1, \ldots, n$, the previous lemma applied to $Q:=Q_{1} \cdots Q_{n}$ yields some $y \in K$ with $P(y)=0$ and $Q_{i}(y) \neq 0$ for all $i=1, \ldots, n$. Now use compactness.

Proof of Proposition 5.4. We can assume that $S:=\mathrm{Z}(P)$ is infinite. The preceding lemma yields an element $a$ in an elementary extension of $K$ with $P(a)=0$ and $Q(a) \neq 0$ for all $Q \in K\left[Y, Y^{\prime}\right]^{\neq}$with $\operatorname{deg}_{Y^{\prime}} Q<d:=\operatorname{deg}_{Y^{\prime}} P$. In particular, $a$ is transcendental over $K$. Since $P$ creates a constant, $K\langle a\rangle=K\left(a, a^{\prime}\right)$ has a constant $c \notin C$. We have $c=A(a) / B(a)$ with $A \in K\left[Y, Y^{\prime}\right], \operatorname{deg}_{Y^{\prime}} A<d, B \in$ $K[Y]^{\neq}$. From $c^{\prime}=0$ we get $A^{\prime}(a) B(a)-A(a) B^{\prime}(a)=0$, so

$$
A^{\prime}(Y) B(Y)-A(Y) B^{\prime}(Y)=D(Y) P(Y) \text { in } K\{Y\} \text { with } D \in K[Y] .
$$

Hence for $y \in S$ with $B(y) \neq 0$ we have $(A(y) / B(y))^{\prime}=0$, that is, $A(y) / B(y) \in C$. Thus for $S_{B}:=\{y \in S: B(y) \neq 0\}$ we have a definable map

$$
f: S_{B} \rightarrow C, \quad f(y):=A(y) / B(y) .
$$

Since $c$ is transcendental over $K, a$ is algebraic over $K(c)$, say

$$
F_{0}(c) a^{e}+F_{1}(c) a^{e-1}+\cdots+F_{e}(c)=0,
$$

where $F_{0}, F_{1}, \ldots, F_{e} \in K[Z]$ have no common divisor of positive degree in $K[Z]$. Let $G:=\partial P / \partial Y^{\prime}$ be the separant of $P$. Then $G(a) \neq 0, K\left[a, a^{\prime}, 1 / B(a), 1 / G(a)\right]$ is a differential subring of $K\left(a, a^{\prime}\right)$, and every $y \in S_{B}$ with $G(y) \neq 0$ yields a differential ring morphism

$$
\phi_{y}: K\left[a, a^{\prime}, 1 / B(a), 1 / G(a)\right] \rightarrow K
$$

that is the identity on $K$ with $\phi_{y}(a)=y$; see the subsection on minimal annihilators in $\left[1\right.$, Section 4.1]. Moreover, $c=A(a) / B(a) \in K\left[a, a^{\prime}, 1 / B(a), 1 / G(a)\right]$, and so for $y \in S_{B}$ with $G(y) \neq 0$ we have $\phi_{y}(c)=A(y) / B(y)=f(y)$, so

$$
F_{0}(f(y)) y^{e}+F_{1}(f(y)) y^{e-1}+\cdots+F_{e}(f(y))=0 .
$$

Set $S_{B, G}:=\left\{y \in S_{B}: G(y) \neq 0\right\}$. Then $S \backslash S_{B, G}$ is finite, and the above shows that for all $z \in f\left(S_{B, G}\right)$ we have $\left|f^{-1}(z) \cap S_{B, G}\right| \leqslant e$. Now use Lemma 5.1.

Freitag [6] proves a generalization of Lemma 5.3. Nishioka ([13], see also [11, p. 90]) gives sufficient conditions on irreducible differential polynomials of order 1 to create a constant, involving the concept of "having no movable singularities"; this can be used to give further examples of $P \in K\{Y\}$ of order 1 whose zero set is 
parametrizable by constants. But we do not know whether $\mathrm{Z}(P)$ is parametrizable by constants for every $P \in K\{Y\}$ of order 1 .

Open problems. The definable set

$$
\left\{y \in \mathbb{T}: y y^{\prime \prime}=\left(y^{\prime}\right)^{2}\right\}=\left\{a \mathrm{e}^{b x}: a, b \in \mathbb{R}\right\} \subseteq \mathbb{T}^{2}
$$

is the image of the map $(a, b) \mapsto a \mathrm{e}^{b x}: \mathbb{R}^{2} \rightarrow \mathbb{T}^{2}$, and so by the above negative result this map is not definable in the differential field $\mathbb{T}$. But it is definable in the exponential differential field $(\mathbb{T}$, exp), where exponentiation on $\mathbb{T}$ is taken as an extra primitive. This raises the question whether parametrizability by constants holds in an extended sense where the parametrizing maps are allowed to be definable in ( $\mathbb{T}$, exp). More precisely, if $S \subseteq \mathbb{T}^{n}$ is definable in $\mathbb{T}$ with $\operatorname{dim} S=0$, does there always exist an $m$ and a map $f: \mathbb{R}^{m} \rightarrow \mathbb{T}^{n}$, definable in $\left(\mathbb{T}\right.$, exp), with $S \subseteq f\left(\mathbb{R}^{n}\right)$ ? (It is enough to have this for $n=1$ and $S=\{y \in \mathbb{T}: P(y)=0\}, P \in \mathbb{T}\{Y\}^{\neq}$.)

This is of course related to the issue whether the results in [1, Chapter 16] about $\mathbb{T}$ generalize to its expansion $(\mathbb{T}, \exp )$. In particular, is the structure induced on $\mathbb{R}$ by $(\mathbb{T}$, exp) just the exponential field structure of $\mathbb{R}$ ?

It would be good to know more about the order types of discrete definable subsets of Liouville closed $\omega$-free newtonian $H$-fields $K$. For example, can any such set have order type $\omega$, or more generally, have an initial segment of order type $\omega$ ?

\section{Dimension $0=$ Co-Analyzable Relative to the Constant Field}

Parametrizability by constants was our first guess of the model-theoretic significance of [1, Theorem 16.0.3] which says that a Liouville closed $\omega$-free newtonian $H$-field has no proper differentially-algebraic $H$-field extension with the same constants. As we saw, this guess failed on the set of zeros of $Y Y^{\prime \prime}-\left(Y^{\prime}\right)^{2}$. We subsequently realized that the notion of co-analyzability from [8] fits exactly our situation. Below we expose what we need from that paper, and next we apply it to $\mathbb{T}$.

Co-analyzability. We adopt here the model-theory notations of [1, Appendix B]. Let $\mathcal{L}$ be a first-order language with a distinguished unary relation symbol $C$. For convenience we assume $\mathcal{L}$ is one-sorted. Let $\boldsymbol{M}=(M ; \ldots)$ be an $\mathcal{L}$-structure and let $C^{M} \subseteq M$ (or just $C$ if $M$ is clear from the context) be the interpretation of the symbol $\bar{C}$ in $\boldsymbol{M}$; we assume $C \neq \emptyset$.

Assume $\boldsymbol{M}$ is $\omega$-saturated. Let $S \subseteq M^{n}$ be definable. By recursion on $r \in \mathbb{N}$ we define what makes $S$ co-analyzable in $r$ steps (tacitly: relative to $M$ and $C$ ):

$\left(\mathrm{C}_{0}\right) S$ is co-analyzable in 0 steps iff $S$ is finite;

$\left(\mathrm{C}_{r+1}\right) S$ is co-analyzable in $r+1$ steps iff for some definable set $R \subseteq C \times M^{n}$,

(a) the natural projection $C \times M^{n} \rightarrow M^{n}$ maps $R$ onto $S$;

(b) for each $c \in C$, the section $R(c):=\left\{s \in M^{n}:(c, s) \in R\right\}$ above $c$ is co-analyzable in $r$ steps.

We call $S$ co-analyzable if $S$ is co-analyzable in $r$ steps for some $r$.

Thus in $\left(\mathrm{C}_{r+1}\right)$ the set $R$ gives rise to a covering $S=\bigcup_{c \in C} R(c)$ of $S$ by definable sets $R(c)$ that are co-analyzable in $r$ steps. Of course, the definable set $C^{r} \subseteq M^{r}$ is the archetype of a definable set that is co-analyzable in $r$ steps. Note that if $S$ is co-analyzable in 1 step, then the $\omega$-saturation of $\boldsymbol{M}$ yields for $R$ as in $\left(\mathrm{C}_{1}\right)$ a uniform bound $e \in \mathbb{N}$ such that $|R(c)| \leqslant e$ for all $c \in C$. This $\omega$-saturation gives likewise an automatic uniformity in $\left(\mathrm{C}_{r+1}\right)$ that enables us to extend the notion of 
co-analyzability appropriately to arbitrary $\boldsymbol{M}$ (not necessarily $\omega$-saturated). Before doing this, we mention some easy consequences of the definition above where we do assume $M$ is $\omega$-saturated. First, if the definable set $S \subseteq M^{n}$ is co-analyzable in $r$ steps, then $S$ is co-analyzable in $r+1$ steps: use induction on $r$. Second, if the definable set $S \subseteq M^{n}$ is co-analyzable in $r$ steps, then so is any definable subset of $S$, and the image $f(S)$ under any definable map $f: S \rightarrow M^{m}$. Third, if the definable sets $S_{1}, S_{2} \subseteq M^{n}$ are co-analyzable in $r_{1}$ and $r_{2}$ steps, respectively, then $S_{1} \cup S_{2}$ is co-analyzable in $\max \left(r_{1}, r_{2}\right)$ steps. Finally, if the definable sets $S_{1} \subseteq M^{n_{1}}$ and $S_{2} \subseteq M^{n_{2}}$ are co-analyzable in $r_{1}$ steps and $r_{2}$ steps, respectively, then $S_{1} \times S_{2} \subseteq M^{n_{1}+n_{2}}$ is co-analyzable in $r_{1}+r_{2}$ steps. In any case, the class of co-analyzable definable sets is clearly very robust.

Next we extend the notion above to arbitrary $\boldsymbol{M}$, not necessarily $\omega$-saturated. Let $S \subseteq M^{n}$ be definable. Define an $r$-step co-analysis of $S$ by recursion on $r \in \mathbb{N}$ as follows: for $r=0$ it is an $e \in \mathbb{N}$ with $|S| \leqslant e$. For $r=1$ it is a tuple $(e, R)$ with $e \in \mathbb{N}$ and definable $R \subseteq C \times M^{n}$ such that the natural projection $C \times M^{n} \rightarrow M^{n}$ maps $R$ onto $S$, and $|R(c)| \leqslant e$ for all $c \in C$. Given $r \geqslant 1$, an $(r+1)$-step co-analysis of $S$ is a tuple $\left(e, R_{1}, \ldots, R_{r+1}\right)$ with $e \in \mathbb{N}$ and definable sets

$$
R_{i} \subseteq C \times M^{n} \times M^{d_{i}} \times \cdots \times M^{d_{r}} \quad\left(i=1, \ldots, r+1, d_{1}, \ldots, d_{r} \in \mathbb{N}\right),
$$

(so $R_{r+1} \subseteq C \times M^{n}$ ), such that the natural projection $C \times M^{n} \rightarrow M^{n}$ maps $R_{r+1}$ onto $S$, and for each $c \in C$ there exists $b \in M^{d_{r}}$ for which the tuple $\left(e, R_{1}^{b}, \ldots, R_{r}^{b}\right)$ is an $r$-step co-analysis of $R_{r+1}(c) \subseteq S$. (Here we use the following notation for a relation $R \subseteq P \times Q$ : for $q \in Q$ we set $R^{q}:=\{p \in P:(p, q) \in R\}$.)

For model-theoretic use the reader should note the following uniformity with respect to parameters from $M^{m}$ : let $e, R_{1}, \ldots, R_{r+1}, S$ be given with $e \in \mathbb{N}, 0$ definable $R_{i} \subseteq M^{m} \times C \times M^{d_{i}} \times \cdots \times M^{d_{r}}$ for $i=1, \ldots, r+1$, and 0-definable $S \subseteq M^{m} \times M^{n}$. Then the set of $a \in M^{m}$ such that $\left(e, R_{1}(a), \ldots, R_{r+1}(a)\right)$ is an $(r+1)$-step co-analysis of $S(a)$ is 0 -definable. Moreover, one can take a defining $\mathcal{L}$ formula for this subset of $M^{m}$ that depends only on $e$ and given defining $\mathcal{L}$-formulas for $R_{1}, \ldots, R_{r+1}, S$, not on $\boldsymbol{M}$.

If $M$ is $\omega$-saturated, then a definable set $S \subseteq M^{n}$ can be shown to be co-analyzable in $r$ steps iff there exists an $r$-step co-analysis of $S$. (To go from co-analyzable in $r$ steps to an $r$-step co-analysis requires the uniformity noted above.) Thus for arbitrary $\boldsymbol{M}$ and definable $S \subseteq M^{n}$ we can define without ambiguity $S$ to be coanalyzable in $r$ steps if there exists an $r$-step co-analysis of $S$; likewise, $S$ is defined to be co-analyzable if $S$ is co-analyzable in $r$ steps for some $r$. After the proof of Lemma 6.3 we give an example of a definable $S \subseteq \mathbb{T}$ that is co-analyzable in 2 steps but not in 1 step (relative to $\mathbb{T}$ and $\mathbb{R}$ ).

Let $S \subseteq M^{n}$ be definable and $\boldsymbol{M}^{*}$ an elementary extension of $\boldsymbol{M}$. We denote by $S^{*} \subseteq\left(M^{*}\right)^{n}$ the extension of $S$ to $\boldsymbol{M}^{*}$ : choose an $\mathcal{L}_{M}$-formula $\varphi(x)$, where $x=$ $\left(x_{1}, \ldots, x_{n}\right)$, with $S=\varphi^{M}$, and set $S^{*}:=\varphi^{M^{*}}$. Then for a tuple $\left(e, R_{1}, \ldots, R_{r+1}\right)$ with $e, r \in \mathbb{N}$ and definable $R_{i} \subseteq C \times M^{n} \times M^{d_{i}} \times \cdots \times M^{d_{r}}$ for $i=1, \ldots, r+1$ we have: $\left(e, R_{1}, \ldots, R_{r+1}\right)$ is an $(r+1)$-step co-analysis of $S$ iff $\left(e, R_{1}^{*}, \ldots, R_{r+1}^{*}\right)$ is an $(r+1)$-step co-analysis of $S^{*}$. Here is [8, Proposition 2.4]:

Proposition 6.1. Let the language $\mathcal{L}$ be countable and let $T$ be a complete $\mathcal{L}$-theory such that $T \vdash \exists x C(x)$. Then the following conditions on an $\mathcal{L}$-formula $\varphi(x)$ with $x=\left(x_{1}, \ldots, x_{n}\right)$ are equivalent: 
(i) for some model $\boldsymbol{M}$ of $T, \varphi^{\boldsymbol{M}}$ is co-analyzable;

(ii) for every model $\boldsymbol{M}$ of $T, \varphi^{\boldsymbol{M}}$ is co-analyzable;

(iii) for every model $\boldsymbol{M}$ of $T$, if $C^{\boldsymbol{M}}$ is countable, then so is $\varphi^{\boldsymbol{M}}$;

(iv) for all models $\boldsymbol{M} \preccurlyeq \boldsymbol{M}^{*}$ of $T$, if $C^{\boldsymbol{M}}=C^{\boldsymbol{M}^{*}}$, then $\varphi^{\boldsymbol{M}}=\varphi^{\boldsymbol{M}^{*}}$.

The equivalence (i) $\Leftrightarrow$ (ii) and the implication (ii) $\Rightarrow$ (iii) are clear from the above, and (iii) $\Rightarrow$ (iv) holds by Vaught's two-cardinal theorem [9, Theorem 12.1.1]. The contrapositive of (iv) $\Rightarrow$ (i) is obtained in [8] by an omitting types argument.

Application to $\mathbb{T}$. Let $\mathcal{L}$ be the language of ordered valued differential fields from Section 3, except that we consider it as having in addition a distinguished unary relation symbol $C$; an $H$-field is construed as an $\mathcal{L}$-structure as before, with $C$ in addition interpreted as its constant field.

Let $K$ be a Liouville closed $\omega$-free newtonian $H$-field and $P \in K\{Y\}^{\neq}$. If $K \preccurlyeq K^{*}$ and $K$ and $K^{*}$ have the same constants, then $P$ has the same zeros in $K$ and $K^{*}$, by [1, Theorem 16.0.3]. Thus the zero set $\mathrm{Z}(P) \subseteq K$ is co-analyzable by Proposition 6.1 applied to the $\mathcal{L}_{A}$-theory $T:=\operatorname{Th}\left(K_{A}\right)$ where $A$ is the finite set of nonzero coefficients of $P$. In fact:

Proposition 6.2. Let $S \subseteq K^{n}$ be definable, $S \neq \emptyset$. Then

$$
S \text { is co-analyzable } \Longleftrightarrow \operatorname{dim} S=0 \text {. }
$$

Proof. Suppose $\operatorname{dim} S=0$. Then for $i=1, \ldots, n$ and the $i$ th coordinate projection $\pi_{i}: K^{n} \rightarrow K$ we have $\operatorname{dim} \pi_{i}(S)=0$, and thus $\pi_{i}(S) \subseteq \mathrm{Z}\left(P_{i}\right)$ with $P_{i} \in K\{Y\}^{\neq}$. Since each $\mathrm{Z}\left(P_{i}\right)$ is co-analyzable and $S \subseteq \mathrm{Z}\left(P_{1}\right) \times \cdots \times \mathrm{Z}\left(P_{n}\right)$, we conclude that $S$ is co-analyzable. Conversely, assume that $S$ is co-analyzable, say in $r$ steps. To get $\operatorname{dim} S=0$ we can arrange that $K$ is $\omega$-saturated. Using $\operatorname{dim} C=0$ and induction on $r$ it follows easily from the behavior of dimension in definable families (Theorem 0.1) that $\operatorname{dim} S=0$.

Let $\operatorname{dim}_{C} S$ be the least $r \in \mathbb{N}$ such that $S$ is co-analyzable in $r$ steps, for nonempty definable $S \subseteq K^{n}$ with $\operatorname{dim} S=0$ (and $\operatorname{dim}_{C} \emptyset:=-\infty$ ). It is easy to show that $\operatorname{dim}_{C} S$ coincides with the usual semialgebraic dimension of $S$ (with respect to the real closed field $C$ ) when $S \subseteq C^{n}$ is semialgebraic. In general, $\operatorname{dim}_{C} S$ behaves much like a dimension function, and it would be good to confirm this by showing for example that for definable $S_{i} \subseteq K^{n_{i}}$ with $\operatorname{dim} S_{i}=0$ for $i=1,2$ we have

$$
\operatorname{dim}_{C} S_{1} \times S_{2}=\operatorname{dim}_{C} S_{1}+\operatorname{dim}_{C} S_{2} .
$$

(We do know that the quantity on the left is at most that on the right.) Another question is whether $\operatorname{dim}_{C} \mathrm{Z}(P) \leqslant \operatorname{order}(P)$ for $P \in K\{Y\}^{\neq}$.

Towards the uniform finiteness property mentioned at the end of the introduction, we introduce a condition that is equivalent to co-analyzability.

Let $K$ be $\omega$-saturated and $S \subseteq K^{n}$ be definable. By recursion on $r \in \mathbb{N}$ we define what makes $S$ fiberable by $C$ in $r$ steps: for $r=0$ it means that $S$ is finite; $S$ is fiberable by $C$ in $(r+1)$ steps iff there is a definable map $f: S \rightarrow C$ such that $f^{-1}(c)$ is fiberable by $C$ in $r$ steps for every $c \in C$.

Lemma 6.3. $S$ is co-analyzable in $r$ steps iff $S$ is fiberable by $C$ in $r$ steps.

Proof. By induction on $r$. The case $r=0$ is trivial. Assume $S$ is co-analyzable in $(r+1)$ steps, so we have a definable $R \subseteq C \times K^{n}$ that is mapped onto $S$ under the natural projection $C \times K^{n} \rightarrow K^{n}$ and such that $R(c)$ is co-analyzable in $r$ steps 
for all $r$. For $s \in S$ the definable nonempty set $R^{s} \subseteq C$ is a finite union of intervals and points, and so we can pick a point $f(s) \in R^{s}$ such that the resulting function $f: S \rightarrow C$ is definable. Then $f^{-1}(c) \subseteq R(c)$ is co-analyzable in $r$ steps for all $c \in C$, and so fiberable by $C$ in $r$ steps by the inductive assumption. Thus $f$ witnesses that $S$ is fiberable by $C$ in $(r+1)$ steps. The other direction is clear.

As an example, consider $S=\mathrm{Z}\left(Y Y^{\prime \prime}-\left(Y^{\prime}\right)^{2}\right)$. Then we have a definable (surjective) function $f: S \rightarrow C$ given by $f(y)=y^{\dagger}$ for nonzero $y \in S$, and $f(0)=0$. For $c \in C^{\times}$ we take any $y \in S$ with $f(y)=c$, and then $f^{-1}(c)=C^{\times} y$; also $f^{-1}(0)=C$. Thus $f$ witnesses that $S$ is fiberable by $C$ in two steps. Moreover, $S$ is not fiberable by $C$ in one step: if it were, Lemma 5.1 would make $S$ parametrizable by constants, which we know is not the case.

An advantage of fiberability by $C$ over co-analyzability is that for $f: S \rightarrow C$ and $R \subseteq C \times S$ witnessing these notions the fibers $f^{-1}(c)$ in $S=\bigcup_{c} f^{-1}(c)$ are pairwise disjoint, which is not necessarily the case for the sections $R(c)$ in $S=\bigcup_{c} R(c)$. Below we use the equivalence

$$
S \text { is finite } \Longleftrightarrow f(S) \text { is finite and every fiber } f^{-1}(c) \text { is finite. }
$$

to obtain the uniform finiteness property mentioned at the end of the introduction. We state this property here again in a slightly different form, with $K$ any Liouville closed $\omega$-free newtonian $H$-field:

Proposition 6.4. Let $D \subseteq K^{m}$ and $S \subseteq D \times K^{n}$ be definable. Then there exists an $e \in \mathbb{N}$ such that $|S(a)| \leqslant e$ whenever $a \in D$ and $S(a)$ is finite.

Proof. We first consider the special case that $n=1$ and $S(a) \subseteq C$ for all $a \in D$. By $[1,16.0 .2($ ii) $]$ a subset of $C$ is definable in $K$ iff it is semialgebraic in the sense of $C$. Thus $S(a)$ is finite iff it doesn't contain any interval $(b, c)$ in $C$ with $b<c$ in $C$; the uniform bound then follows by a routine compactness argument. Next we reduce the general case to this special case.

First, using Proposition 1.6 we shrink $D$ to arrange that $\operatorname{dim} S(a)=0$ for all $a \in D$. Next, we arrange that $K$ is $\omega$-saturated, so $S(a)$ is fiberable by $C$ for every $a \in D$. Saturation allows us to reduce further to the case that for a fixed $r \in \mathbb{N}$ every section $S(a)$ is fiberable by $C$ in $(r+1)$ steps. We now proceed by induction on $r$. Model-theoretic compactness yields a definable function $f: S \rightarrow C$ such that for every $a \in D$ the function $f_{a}: S(a) \rightarrow C$ given by $f_{a}(s)=f(a, s)$ witnesses that $S(a)$ is fiberable by $C$ in $(r+1)$ steps, that is, $f_{a}^{-1}(c)$ is fiberable by $C$ in $r$ steps for all $c \in C$.

Inductively we have $e \in \mathbb{N}$ such that $\left|f_{a}^{-1}(c)\right| \leqslant e$ whenever $a \in D, c \in C$, and $f_{a}^{-1}(c)$ is finite. The special case we did in the beginning of the proof gives $d \in \mathbb{N}$ such that $\left|f_{a}(S(a))\right| \leqslant d$ whenever $a \in D$ and $f_{a}(S(a))$ is finite. For $a \in D$ we have $S(a)=\bigcup_{c} f_{a}^{-1}(c)$, so if $S(a)$ is finite, then $|S(a)| \leqslant d e$.

To fully justify the use of saturation/model-theoretic compactness in the proof above requires an explicit notion of " $r$-step fibration by $C$ " (analogous to that of " $r$-step co-analysis") that makes sense for any $K$, not necessarily $\omega$-saturated. We leave this to the reader, and just note a nice consequence: if $S \subseteq K^{n}$ is definable, infinite, and $\operatorname{dim} S=0$, then $S$ has the same cardinality as $C$. (This reduces to the fact that any infinite semialgebraic subset of $C$ has the same cardinality as $C$.) In particular, there is no countably infinite definable set $S \subseteq \mathbb{T}$. 
As an application of the material above we show that the differential field $K$ does not eliminate imaginaries. More precisely:

Corollary 6.5. No definable map $f: K^{\times} \rightarrow K^{n}$ is such that for all $a, b \in K^{\times}$,

$$
a \asymp b \Longleftrightarrow f(a)=f(b) \text {. }
$$

Proof. By [1, Lemmas 16.6.10, 14.5.10] there exists an elementary extension of $K$ with the same constant field $C$ as $K$ and whose value group has greater cardinality than $C$. Suppose $f: K^{\times} \rightarrow K^{n}$ is definable such that for all $a, b \in K^{\times}$we have: $a \asymp b \Leftrightarrow f(a)=f(b)$. We can arrange that the value group of $K$ has greater cardinality than $C$, and so $f\left(K^{\times}\right) \subseteq K^{n}$ has dimension $>0$. Every fiber $f^{-1}(p)$ with $p \in f\left(K^{\times}\right)$is a nonempty open subset of $K^{\times}$, so has dimension 1 , and thus $\operatorname{dim} K^{\times}>1$ by d-boundedness of $K$, a contradiction.

\section{REFERENCES}

[1] M. Aschenbrenner, L. van den Dries, J. van der Hoeven, Asymptotic Differential Algebra and Model Theory of Transseries, Ann. of Math. Stud., to appear, arXiv:1509.02588.

$[2]$, The group of strong automorphisms of the differential field of transseries, in preparation.

[3] T. Brihaye, C. Michaux, C. Rivière, Cell decomposition and dimension function in the theory of closed ordered differential fields, Ann. Pure Appl. Logic 159 (2009), no. 1-2, 111-128.

[4] L. van den Dries, Dimension of definable sets, algebraic boundedness and Henselian fields, Ann. Pure Appl. Logic 45 (1989), no. 2, 189-209.

[5] L. van den Dries, A. Macintyre, D. Marker, Logarithmic-exponential series, Ann. Pure Appl. Logic 111 (2001), 61-113.

[6] J. Freitag, Disintegrated order one differential equations and algebraic general solutions, preprint, 2016, arXiv:1607.04387.

[7] N. Guzy, F. Point, Topological differential fields and dimension functions, J. Symbolic Logic 77 (2012), no. 4, 1147-1164.

[8] B. Herwig, E. Hrushovski, D. Macpherson, Interpretable groups, stably embedded sets, and Vaughtian pairs, J. London Math. Soc. (2003) 68, no. 1, 1-11.

[9] W. Hodges, Model Theory, Encyclopedia of Mathematics and its Applications, vol. 42, Cambridge University Press, Cambridge, 1993.

[10] J. Johnson, Systems of $n$ partial differential equations in $n$ unknown functions: the conjecture of M. Janet, Trans. Amer. Math. Soc. 242 (1977), 329-334.

[11] M. Matsuda, First Order Algebraic Differential Equations: A Differential Algebraic Approach, Lecture Notes in Math., vol. 804, Springer-Verlag, Berlin-Heidelberg, 1980.

[12] T. McGrail, The search for trivial types, Illinois J. Math. 44 (2000), no. 2, 263-271.

[13] K. Nishioka, Algebraic differential equations of Clairaut type from the differential-algebraic standpoint, J. Math. Soc. Japan 31 (1979), 191-197.

[14] A. Pillay, Around differential Galois theory, in: M. A. H. MacCallum, A. V. Mikhailov (eds.), Algebraic Theory of Differential Equations, pp. 232-240, London Mathematical Society Lecture Note Series, vol. 357, Cambridge University Press, Cambridge, 2009.

[15] M. Rosenlicht, The nonminimality of the differential closure, Pacific J. Math. 52 (1974), 529-537.

Department of Mathematics, University of California, los Angeles, Los Angeles, CA 90095, U.S.A.

E-mail address: matthias@math.ucla.edu

Department of Mathematics, University of Illinois at Urbana-Champaign, Urbana, IL 61801 , U.S.A.

E-mail address: vddries@math.uiuc.edu

École Polytechnique, 91128 Palaiseau Cedex, France

E-mail address: vdhoeven@lix.polytechnique.fr 